\title{
Polarization of Infrared Objects
}

\author{
A. KRUSZEWSKI (Warsaw) \\ (read by J. SMAK, Warsaw)
}

We know several infrared objects showing large intrinsic polarization (FORBES 1967, APPENZELLER and O'DELL 1967, KRUSZEWSKI 1968, SERKOWSKI 1969, SHAWL 1969, SHAWL and ZELLNER 1970, KRUSZEWSKI 1971, DYCK, FORBES and SHAWL 1971).

In the following discussion I shall speak about the broad category of infrared stars which consists of reddest and coolest giants ( $\mathrm{M}_{8}$ or later) and supergiants ( $\mathrm{M}_{5}$ or later). This restriction is made because the yellow supergiants seem to have different polarization properties and these properties most likely extend to supergiants as late as $\mathrm{M} 2$ or even M4. Also it is not clear if normal red variables can be treated together with infrared objects.

The most striking polarization property of infrared objects is the fact that almost all of them show large polarization in the optical infrared. It should be recalled that only small proportion of red variables have appreciable polarization and in addition this polarization is usually very small around $1 \mu$. Only for few extreme cases the polarization in this spectral region attains 1 or $2 \%$ (KRUSZEWSKI, GEHRELS and SERKOWSKI 1968). Out of 12 infrared objects observed for polarization in the optical infrared only two (CIT 8 and CIT 14) failed to show polarization larger than $2 \%$. In few cases the polarization might be of the interstellar origin but most often the high galactic latitude and/or time variability of the polarization points on the intrinsic nature of the observed polarization.

In all cases that the polarization was measured at shorter wavelengths and it is not dominated by the interstellar contribution (NML Tau, CIT 6, VY CMa and IRC +10216) the wavelength dependence is steep like in red variables and in all cases (including NML Cyg and CIT 10) attains $10 \%$ or more. The object CIT 8 may be the only exception if we rely on a single observation taken around the maximum of brightness.

In two cases that the polarization was observed during appreciable changes of brightness (NML Tau and CIT 6) the polarization was the smallest at the maximum brightness when the color is also the bluest. IRC +10216 may also show this type of relation between the polarization and brightness (DYCK et al. 1971).

We have two concurrent explanations for the origin of the intrinsic polarization in cool variables. The first is based on an asymmetric envelope with atoms, molecules or refractory grains scattering the light emerging from the photosphere. The second assumes that the polarization is produced by the aligned absorbing particles. Up to now we have evidences (DYCK and SANDFORD 1971, DYCK et al. 1971, DERVIZ and DOMBROVSKY 1971) that the grains are the more likely possibility than the molecules. In addition the source of polarization seems to lie in the circumstellar envelope and not in the photosphere.

Models of the scattering envelope (KRUSZEWSKI et al. 1968, ANGEL 1969) give a conclusion that the produced polarization can be barely exceeding $5 \%$. Higher polarizations can result only when the envelope is of irregular structure with a thick cloud situated in front of the star. Let us assume that in all observed cases the polarization is produced by scattering. Then for each group of highly polarized objects with specific thickness and geometry of the envelope we should observe even larger number of objects possessing the same envelope but showing small polarization. We have seen that within a class of the infrared objects all sufficiently well observed stars show high polarizations. However we can have more precise informations about the importance of envelope. The infrared color $\mathrm{L}-\mathrm{O}$ can be used for that purpose. (GILLETT, MERRILL and STEIN 1971. DYCK et al. 1971, GEHRZ and WOOLF 1971). 


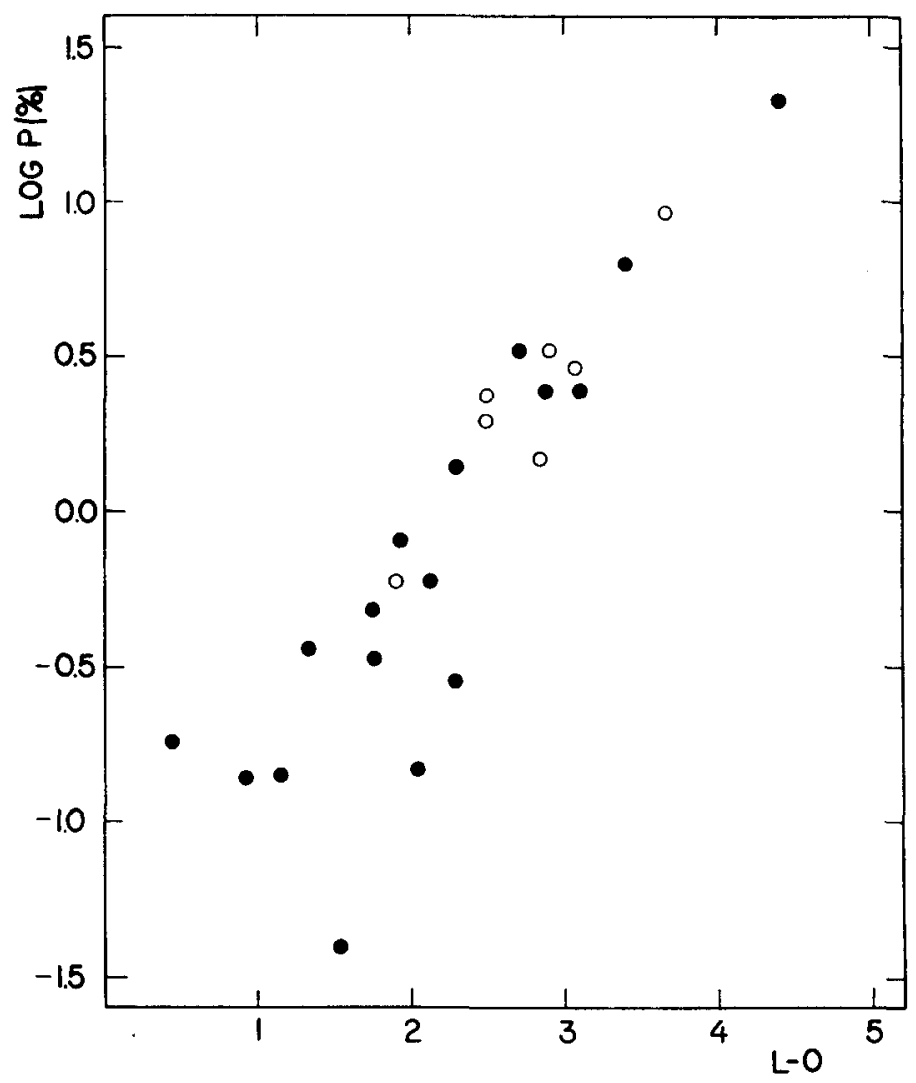

Fig. 1: The logarithm of the degree of polarization at $\lambda=9000 \AA$ plotted against the infrared color $\mathrm{L}-\mathrm{O}$. For cases where the interstellar contribution to the polarization may be important the open circles are used. Only $M$ type and carbon stars are shown.

Fig. 1 shows a plot of the logarithm of the degree of polarization against the $\mathrm{L}-\mathrm{O}$ color. This figure was used by DYCK et al. (1971) for demonstrating the close relation between the polarization and the infrared excess. Here we use the polarization in the optical infrared instead of the visual polarization what enlarges the number of the infrared objects available for statistics. The open circles denote objects for which the interstellar contribution can be important. Only $M$ type and carbon stars are plotted. We can see that for few most extreme polarizations there are no objects with the small polarization and equally large infrared excess. Only if we enlarge our sample by adding the yellow supergiants of $\mathrm{K}$ type we find objects with largest infrared excesses and relatively small polarizations (SERKOWSKl 1971). Therefore only the unlikely possibility that the star has different spectral type and different characteristic period of brightness variations depending on aspect can save the scattering hypothesis. Hence we are left with a tentative conclusion that for the infrared objects the aligned grain absorption or some other unknown mechanism is operating. 
For aligment of particles we need the stellar magnetic field of sufficient strength. In case of the DAVIS-GREENSTEIN mechanisms of orientation of particles (JONES and SPITZER 1967, GREENBERG 1968) we have the relaxation time for orientation

$$
\tau_{0}=\frac{o \mathrm{I}}{\chi^{\prime \prime} \mathrm{VB}},
$$

where $\omega$ is the root-mean-square angular velocity of the grain, I is the average moment of inertia, $\chi^{\prime \prime}$ is the imaginary part of the magnetic susceptibility, $\mathrm{V}$ is the volume of the grain and $\mathrm{B}$ is the magnetic field strength. The relaxation time for collisions $\tau_{\mathrm{e}}$ is

$$
\tau_{\mathrm{c}}=\frac{\mathrm{m}_{\mathrm{g}}}{\mathrm{n}_{\mathrm{H}} A v_{\mathrm{H}} \mathrm{n}_{\mathrm{H}}} \text {, }
$$

where $\mathrm{m}_{\mathrm{g}}$ is the mass of the grain, $\mathrm{m}_{\mathrm{H}}$ is the mass of hydrogen atom, $A$ is the average cross-sectional area of the grain, $v_{H}$ is the average velocity of the hydrogen aton and $\mathbf{n}_{\mathrm{H}}$ is the number density of the hydrogen atoms.

After equalizing these two relaxation times we obtain the minimum magnetic field needed for grain orientation

$$
\begin{aligned}
\mathrm{B}^{2} & =10^{-8} \mathrm{a} \mathrm{T}^{1 / 2} \mathrm{n}_{\mathrm{H}} \mathrm{T}_{\mathrm{g}} \quad \text { (dielectric particles) } \\
& =3 \times 10^{-9} \mathrm{a} \mathrm{T} \mathrm{T}^{1 / 2} \mathrm{n}_{\mathrm{H}} T_{\mathrm{G}}^{2} \quad \text { (graphite) }
\end{aligned}
$$

where $T_{g}$ is the temperature of the grain, $T$ is the gas temperature, $a$ is the average radius of the grain, and the magnetic field strength $\mathrm{B}$ is expressed in gauss.

We shall adopt the following physical conditions for a region of silicate grains formation (JENNINGS and DYCK 1971, FIX 1971) $\mathrm{T}=1.5 \times 10^{3 \circ} \mathrm{K}, \mathrm{T}_{\mathrm{g}}=10^{\circ}{ }^{\circ} \mathrm{K}, \mathrm{P}=10 \mathrm{dyn}$, $\mathrm{n}_{\mathrm{H}}=10^{14} \mathrm{~cm}^{-3}, a=7 \times 10^{-6} \mathrm{~cm}$. For such conditions the minimum magnetic field is $B \approx 500$ gauss. The region of grain formation is relatively low above the photosphere so the derived magnetic field strength is practically the same as the photospheric value which can be directly observed.

Moving outward the strength of the dipole magnetic field goes down like $\mathrm{r}^{-3}$ where $\mathrm{r}$ is the distance from the center of tive star. The number density of the gas particles at first goes down very fast up to the point where the expanding motion is effectively started, and here we have the optimal conditions for the magnetic alignment of the dust particles. From this point on the number density of the gas goes down like $\mathrm{r}^{-2}$ and therefore the conditions for the alignment get less favorable. But even the conservative estimate $\mathrm{B}=500$ gauss is not in a serious conflict with the observations. BABCOCK $(1960)$ has measured such magnetic fields in few red variables. For such a crude estimate the agreement can be considered satisfactory.

$$
\text { References: }
$$

ANGEL, J. R. P., Ap. J. 158, 219 (1969).

APPENZELLER, I., and O'DELL, C. R., Ap. J. Letters 149, I5 (1967).

BABCOCK, H. W., in "Stars and Stellar Systems“" vol. 6, ed. J. L. GRELNSTEIN (Chicago: University of Chicago Press) p. 282 (1960).

DERVIZ, T. E., and DOMBPOVSKY, V. A., Astron. Tsirc. 607, 2 (1971).

DYCK, H. M., FORREST, W. J.. GILLETT, F. C., STEIN, W. A., GEHR7, R. D., WOOLF, N. J., and SHAWL, S. J., Ap. J. 165, 57 (1971).

DYCK, H. M., FORBES, F. F., and SHAWL, S. J., preprint (1971).

FIX, J. D., Kitt Peak Contr. No. 554, 213 (1971).

FORBES, F. F., Ap. J. 147, 1226 (1967).

GEHRZ, R. D., and WOOLF, N. J., Ap. J. 165, 285 (1971).

GILLETT, F. C., MERRILI, K. M., and STE]N, W. A., Ap. J. 164, 83 (1971).

GREENBERG, J. M., in "Stars and Stellar Systems" vol. 7, ed. B. M. MIDDIEHURST and I. IT. ALT_ER (Chicago:"University of Chicago Press), p. 221 (1968).

JENNINGS, M. C., and DYCK, H. M., Kitt Peak Contr. No. 554, 203 (1971).

JONES, R. V., and SPITZER, L., Ap. J. 147, 943 (1967).

KRUSZEWSKI, A., P.A.S.P. 80, 560 (1968).

KRUSTEWSKI, A., A. J. 76, 576 (1971).

KRUSZEWSKI, A., GEHRELS, T., and SIRRKOWSKI, K., A.J. 73, 677 (1968).

SERKOWSKI, K., Ap. J. Letters 156, L139 (1969).

SERKOWSKI, K., Kitt Peak Contr. No. 554, 107 (1971).

SHAWL, S. J., Ap. J. Letters 157, L57 (1969).

SHAWI, S. J., and ZELLNER, B., Ap. J. Letters 162, L19 (1970). 